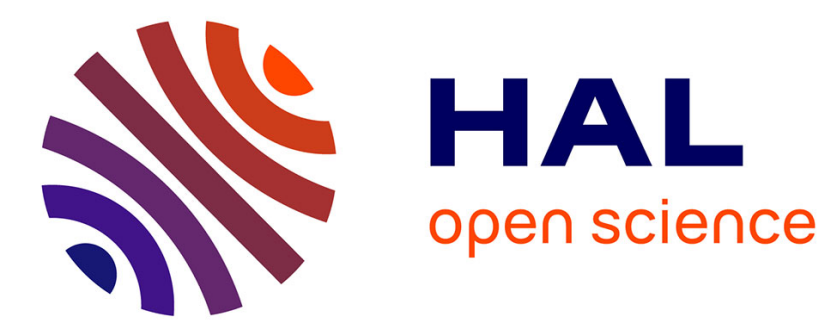

\title{
Methodological Introduction of Context Awareness in the Design of 3GPP Conversational Services
}

\author{
François Toutain, Ahmed Bouabdallah, Radim Zemek, Claude Daloz
}

\section{To cite this version:}

François Toutain, Ahmed Bouabdallah, Radim Zemek, Claude Daloz. Methodological Introduction of Context Awareness in the Design of 3GPP Conversational Services. NGMAST 2012: 6th International Conference on Next Generation Mobile Applications, Services and Technologies, Sep 2012, Paris, France. pp.112-117. hal-00924479

\section{HAL Id: hal-00924479 https://hal.science/hal-00924479}

Submitted on 6 Jan 2014

HAL is a multi-disciplinary open access archive for the deposit and dissemination of scientific research documents, whether they are published or not. The documents may come from teaching and research institutions in France or abroad, or from public or private research centers.
L'archive ouverte pluridisciplinaire HAL, est destinée au dépôt et à la diffusion de documents scientifiques de niveau recherche, publiés ou non, émanant des établissements d'enseignement et de recherche français ou étrangers, des laboratoires publics ou privés. 


\section{Methodological Introduction of Context Awareness in the Design of 3GPP Conversational Services}

\author{
François Toutain \\ Orange Labs Lannion \\ France Telecom R\&D \\ Lannion, France \\ francois.toutain@orange.com
}

\author{
Ahmed Bouabdallah \\ Telecom Bretagne \\ Institut Mines-Telecom \\ Cesson-Sévigné, France \\ ahmed.bouabdallah@telecom- \\ bretagne.eu
}

\author{
Radim Zemek \\ Orange Labs Tokyo \\ France Telecom R\&D \\ Tokyo, Japan \\ radim.zemek@orange.com
}

\author{
Claude Daloz \\ Orange Labs Lannion \\ France Telecom R\&D \\ Lannion, France \\ claude.daloz@orange.com
}

\begin{abstract}
By complementing basic call services, supplementary services totalling nowadays more than twenty different functions, have a long history crossing the main areas of telecommunications from ISDN via ETSI GSM, ETSI TISPAN to finally end at 3GPP and GSMA. They are commonly used in professional settings while their usage is somewhat less developed in mass market situations. They suffer in fact from serious drawbacks preventing them to be massively adopted. We believe however that the attention toward supplementary services may significantly increase due to the exceptional concomitance of two main trends. The first one concerns the current interest boasted by the introduction of next generation networks, such as 4G/IMS through the MMTEL specification efforts. The second trend is determined by the unprecedented levels reached by the mass market penetration of smartphones putting within reach of majority, the enhanced capabilities of these powerful terminals, able to completely renew the end user experience. We claim that context awareness could be the right catalyst allowing a fruitful combination of these two long-term directions of the telecommunication domain. To work towards this goal, we introduce in this paper a methodology allowing the systematic and comprehensive introduction of context-awareness in conversational services. Our methodology involves four main building blocks, we illustrate through various examples drawn from supplementary services. A taxonomy of conversational services is defined and applied to supplementary services. Pending issues complete the analysis of the methodology.
\end{abstract}

Keywords : Context Awareness, Multimedia Telephony, Supplementary Service, Methodology, Taxonomy.

\section{INTRODUCTION}

Supplementary services such as Call Forwarding on No Reply (CFNR), Call Waiting (CW), or Closed User Group (CUG) complement basic call services [1] and are commonly used in professional settings while their usage is somewhat less developed in mass market situations. Totalling nowadays more than twenty different functions, supplementary services have a long history, coming from legacy ISDN (Integrated Services Digital Networks) [2]. ETSI (European Telecommunications Standards Institute) quickly introduced them in GSM [3] and in the same vein 3GPP (Third Generation Partnership Project) adopted them for the UMTS architectures without major evolution until Release 6. Adaptation of these features to IMS (IP Multimedia Subsystem) and NGN (Next Generation
Network) was first pioneered by ETSI TISPAN (Telecom and Internet Converged Services and Protocols for Advanced Networks) [4]. 3GPP taken up part of ETSI works began in Release 7 the development of MMTel (Multimedia Telephony service) [5]. 3GPP has afterwards been entrusted the mission to continue the unified and coherent development of MMTel together with common IMS. Last but not least supplementary services held also attention of GSMA (GSM Association) which introduces part of them in its work concerning the definition of an IMS Profile for Voice and SMS [6].

It is no surprise that these services have been specified through a large standardization body. Indeed, standardization processes are of critical importance for interoperability issues and consistent behaviour leading to wide adoption. That's why basic telecommunication services benefited of such a huge standardization effort. But, although they are very widely known, supplementary services are not really massively adopted as it has been the case for example about SMS (Short Message Service) or mobility, except for the professional area where the specific needs of large and small businesses have led to a marginally wider use. The reasons of such a situation may be quite complex; it appears however that the poor quality of experience emerging from the usage of these supplementary services, constitutes one of its main explanatory factor. Let's first remark that past implementations of these features as well as current ones offer very mediocre dial-based interfaces, which make them difficult and awkward to use [7]. They exhibit very limited and rigid behaviour. And due finally to the "walled-garden networks" principle governing network operators' policies, these features are devoid of any customization capability. Last but not least, the feature interaction problem, which is a quite complicated matter far from being completely solved, often precludes the simultaneous use of these various service elements [8].

With all these drawbacks, it is no surprise that network operators around the world have hardly promoted the majority of supplementary services, and have fallen back on simple strategies such as default provisioning within packaged subscription plans. However, we believe that the attention toward supplementary services may soon increase significantly. Indeed, there is a current interest boasted by the introduction of next generation networks, such as 4G/IMS (IP Multimedia Subsystem), through the MMTEL specification efforts [5]. We can also quote as a recent trend the RCS 
initiative (Rich Communication Suite) which aimed at large audiences, and was however reduced later on to the RCS-e [9] (basically because of cost structures and thus a requirement to reduce the introduction cost of these new technologies). In addition, the mass market penetration of smartphones is reaching unprecedented levels, and these powerful terminals, equipped with large screens are beginning to offer renewed basic services, taking advantage of the smartphone capabilities, such as the visual voicemail feature (provided eg. on Apple's iPhone or Android-based mobile terminals).

Our thesis in this paper is that these increased capabilities, available on smartphone-class terminals for an increasing number of subscribers, are an opportunity to provide supplementary services which will be easier to configure and use. Indeed, these capabilities are key to offer a better user experience, and so far, this has been almost exclusively the case for over-the-top services, implemented by third parties having nothing in common with network operators, while the classical communication services have been kept to a very basic level of functionality. This evolution has leaded to the current situation, where there is a clear dichotomy between advanced services which benefit from capabilities of new platforms but are not standardized, leading to fragmentation, and classical services, standardized but lacking from improved user experience.

In an attempt to improve the situation without restricting the market to high end terminals, the RCS initiative considered ambitious features such as an enhanced phonebook, enriched communications with content sharing capabilities, and enhanced messaging services. It was however reduced to RCSe which focuses on capability sharing, and basically ignored classical supplementary services [9]. MMTEL which could appear as the standard side of RCS, relies on the NGN 's generalized conversational session between mobile or/and fixed users involving various media (speech, video, text, ...); like RCS-e MMTEL introduces sharing capabilities but differently from it, authorizes an enrichment of a session through the execution of updated versions of supplementary services [5][10] deployed in dedicated SIP application server called TAS (Telephony Application Server) [11].

One interesting approach close to RCS/MMTEL initiatives is that of context awareness. Indeed, context could be one of the ways to make services flexible and adaptive. Context aware systems are defined as ones which can adapt their behaviour depending on changing user situations (context adaptation), and also as systems able to explicitly present context data to the users (context presentation) [12]. Context-awareness has been studied for many years, with strong results, and already some independent services being deployed (e.g. GrandCentral aka Google Voice, OneNumber). We believe that now is the proper time to bring context to core services, because, although these are well-known services, context awareness could well enhance the subscribers' willingness to use them. We also advocate that it is an important matter to push this approach to standardization.

In this paper we analyze existing supplementary services, and we introduce a methodology allowing us to produce context-aware versions of those services. Usage of such context-aware services is illustrated by some use cases. A global taxonomy of conversational services applied to supplementary services concludes the paper together with the analysis of the methodology and the pending issues.

\section{PRESENTATION OF THE METHODOLOGY}

Before exposing our methodology for adding contextawareness to communication services, we must introduce some technical definitions. In this paper, context is defined as soft real-time information pieces related to a user situation, be it some physical measurement, logical status, or a combination of several such data [12]. Context is usually acquired through some low-level systems, e.g. sensors, and/or derived from logical information. Low-level context data may further be processed into higher level information, using a variety of techniques such as logical inference or data fusion. Examples of context data include weather condition, localization, user's availability, current agenda slot, number of people around user, etc.

Our focus in this paper is to define context-aware conversational/interpersonal services, starting from classically defined call services in $3 \mathrm{GPP}$ or equivalently communication services in the MMTEL standardization effort. These services obey a well-defined life-cycle, by which [13] :

- a user subscribes to service S ;

- upon approval, the service operator provisions $\mathrm{S}$, using some predefined data coming in part from the user and in part from the operator itself (these parameters being operator embedded data, freely chosen within the standards constraints in order to match a specified service level) ;

- depending on the service, activation can be performed by the operator after which they are always-on services, or activation can be done bit by bit by the user herself;

- invocation can be implicit (for always-on services), or explicit by the user. In the latter case she can provide some parameters during invocation;

- deactivation can be performed for some services ;

- widthdrawal of the service is the last step.

These services specifically target operator networks, meaning that all the operations occurring in the network are strictly and closely controlled by the operator [14].

Studying the addition of context-awareness to these services can be done in a systematic manner. This is the purpose of the following methodology. 


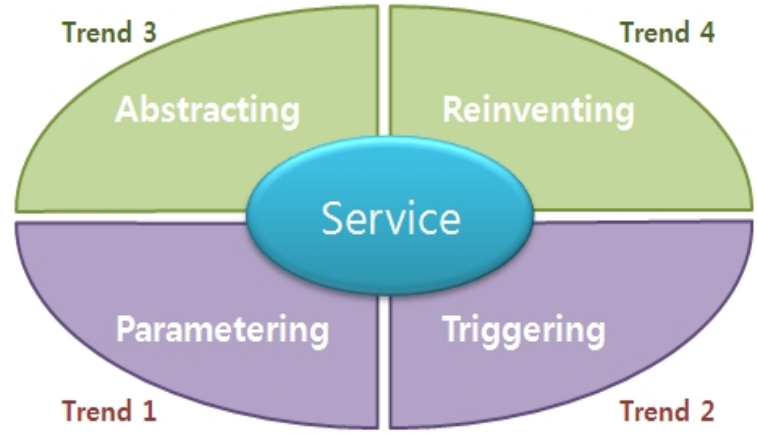

Figure 1. The building blocks of the methodology

Figure 1 depicts four different trends allowing to take context data into account within a service. Trends $1 \& 2$ are about using context awareness to modify the service mechanisms (implying low-level changes to the services in a systematic approach). Trends $3 \& 4$ are about revisiting the services in a more creative way (abstracting the service, or reinventing the service by relaxing some prior assumptions in the initial specification).

Trend 1 is concerned with the service parameters. Conversational services somehow control the calls placed by users, using specific informations provided by the network operator or by the user herself. Static parameters are defined during the service subscription, usually by selecting proper designed values, fixed by the operator at service creation time, are more rarely by the user. Dynamic parameters are input by the user at service invocation time. Context situations can obviously have an influence on these parameters. Previously static parameters can become more dynamic, thanks to changing context data; and dynamic parameters can come from context situations, instead of being input by the user, or in addition to it. An example of static parameter becoming context-aware is the no answer timer in the CFNR feature [15]. In current services instances, operators have to decide on a fixed, good-for-all value for the timer, before the service forwards the incoming call to its new target (e.g. the voicemail system). In a context-aware CFNR feature, the timer can be adapted, for instance, after a learning phase allowing to finetune it to every single user. An example for a dynamic parameter could be the target number of the same service, which could make use of the pro/perso status of the user to change the targeted number, and send private calls to private voicemails whereas professional calls would be routed to a colleague's office.

Trend 2 deals with the service triggering, by which is meant service invocation on the user side, or service activation on the operator's part. In classical implementations, these operations are performed in a static way, so that a service is always-on, or is available on user's request. Using context-awareness, we can change this behaviour, so that a service can be triggered depending on some context condition, which can be very dynamic in nature. As an example, consider the Call Deflection (CD) feature, by which a user terminal is able to avoid the user being interrupted by an incoming call, by deflecting it to another destination [15]. In a classical implementation, the user must activate the $\mathrm{CD}$ service and provide a target identification for the potential bouncing calls. In a context-aware version of this service, users' activity, e.g. driving a car, can be automatically used to infer that users are busy, and the service can be autonomously activated. Conversely, the service triggering can also be inhibited due to context conditions. An example is an emergency call that could get through the $\mathrm{CD}$ service.

Trend 3 is about unifying several services when they can relate to one another thanks to adding some shared context awareness. Indeed, several classical services are very similar except for a specific condition which is explicit in the standards. By relaxing these static condition definitions into a more general context condition, we can unify the services into one, more general, context-aware service. It is interesting to point that 3GPP began to apply this same trend when defining in MMTEL a family of features named Communication Diversion (CDIV) [5][15] bringing the various forwarding features (Unconditional (CFU), on Busy (CFB), on No Reply (CFNR), on Subscriber Not Reachable (CFNRc), on Not Logged-in (CFNL)) together with the Deflection one (CD). It is however worth noting that even if this regrouping is intuitively well-founded, solid arguments firmly founding it, are still missing. We argue in fact that context awareness can provide the right angle to formally define such unification. We will expand this point more thoroughly in the next section.

Trend 4, finally, relates to relaxing even more the original specifications, and allowing ourselves to "re-think" the services with context-awareness in mind. One example of this trend could be an enhanced Multi Level Precedence and Premption service (eMLPP), which is traditionally specified as an alwayson service allowing to preserve a priori defined, important calls, in times of resource scarcity or network congestion [16]. By relaxing this assumption, we can imagine a dynamic service which, by acquiring a context information pertaining to the subject of the call, by way of word recognition for instance, would be able to classify the call importance dynamically, thus preserving truly important calls instead of predefined ones. Obviously such a service would raise some privacy concerns, as is often the case with context-aware systems [17].

\section{Methodology AT WORK}

The main point of trend 1 concerns the contextual derivation of static and/or dynamic parameters used during the invocation of the feature. Lets take CFNR and suppose it has been activated (this is usually the default case in mobile networks [15]). Its main characteristics concerns the ringing duration controlled by a timer, the value of which, instead of being statically fixed by the operator, could be dynamically decreased/increased according to the context situation. We illustrate this point through various use cases associated to specific contextual situations :

- the context allows us to know that the terminal is not in a reasonable proximity to the callee to let the latter answer the call; a predefined ringing will be useless and we can therefore reduce the timer value to its minimum value (to preserve the integrity of the call $\log$, it is necessary that the callee terminal rings at least once). Proximity between the 
callee and her terminal can be inferred from various sources which must be combined : accelerometer data from the mobile phone sensor can indicate if the user is currently carrying it ; location of the terminal associated with user agenda could reveal that they don't share the same place, ....

- the context allows us to know that due to her activity, the user is unable to answer the call, because for instance she is currently driving. In this case, the velocity of the terminal can be obtained from the GPS sensor, allowing to deduce that the user is currently moving at a certain speed. This information is not sufficient and must be combined with additional sources, coming in our example from the car system, which may identify the current driver [18], and consolidated e.g. with map matching, in order to compare the user trajectory with known roads.

- the context allows us to know that the caller is in distress [19], and that her call is implicitly an urgent call. It therefore makes sense, in this case, to extend the ringing duration by increasing the value of the associated timer. A prolonged ringing will be a clear indication to the callee that the call is a special one.

The previous examples manage context information associated to the caller or to the callee. It is interesting to note that when combining both, we may have a service interaction which could be easily solved through priority. Suppose that a caller in distress makes a call to a buddy currently driving. It is reasonable to require that in such a case the no-answer timer duration must be increased (priority to caller situation), to allow the callee to park her car and answer the call.

Trend 2 concerns the dynamical activation of a feature due to specific contextual situation. Suppose the user does not want to be disturbed by a phone call because she is for example at the theater and the context allows us to know this information. It is quite natural when receiving the call, to activate a call deflection feature which will transfer the incoming call to a new address. In our use case, an indoor location system could be used to deduce in an accurate way the right location of the callee; but this information is insufficient and must be combined at least with the exact schedule of the program, to make sure the user cannot currently answer to the call. We note that the value of the new address where to route the deflected call, is a parameter which could also be deduced from the context as explained in trend 1 .

Trend 3 may be well illustrated with the forwarding features family-defined in the standards. It identifies simple situations of the callee preventing her to answer the call : she is currently already engaged in a call (CFB), or does not answer (CFNR), the call cannot be completed due to internal core network routing problems (CFNRc), the callee is not reachable from the radio access network (CFNRc) or the callee has not registered her terminal (CFNL). All these cases clearly are contextual situations of the callee which can be easily managed thanks to the signalling protocol used to establish the call. If we extend to context not deducible from the signalling protocol but from the state of the callee or of the caller, we are led to define contextual call forwarding as the feature with the capability of modifying, in the core network, the management of the call setup, depending on the caller and callee contexts.

Finally, trend 4 in our methodology deals with reinventing the service, or relaxing assumptions and restrictions imposed by the classical service specification. Applying this view to call forwarding services, we notice that the classical descriptions provide for an optional notification service, by way of which the caller can be notified that her call is being rerouted to a new destination [15]. This is a clear opportunity to extend the service family, to include a more useful notification, carrying additional information of a contextual nature. For instance, the notification can provide the caller with a reason for the deflection, along with the target of the redirection, e.g. "Alice is currently driving, you are redirected to her voicemail". The same idea applies to situations where the timer parameter is changed: by providing the caller with a better understanding of the callee's situation, one enhances the chances that both parties end up being in contact. For instance, a call which was classified as an emergency one could lead to the caller receiving a notification similar to "Your correspondent is being informed that your call is urgent, please hold on".

\section{ANALYSIS AND ISSUES}

The methodology introduced above, can be applied to any general conversational service. We however target in this paper the well-known supplementary services which as indicated in the introduction, have a long history crossing the main areas of telecommunications from ISDN via ETSI GSM, ETSI TISPAN to finally end at 3GPP and GSMA. We may in fact consider two main versions of these features depending on whether they are grafted on a CS-based or IP-based telephony service. These two versions are generally close, but they don't necessarily superpose and they furthermore present occasionally tricky semantic differences justifying their distinction [10]. For the sake of clarity, we will in what follows use the term "feature" or "supplementary service" when the underlying bearer doesn't import, and in the other cases :

- "CS-SS" to identify the ones determined by the direct descendants of the initial ISDN supplementary services [1]

- "IMS-SS" to name the ones arising from the adaptation of supplementary services to IMS and NGN [5][20]

Our methodology helped us to develop a comprehensive and systematic introduction of context awareness in all these features. The complete work will deserve by its own a dedicated publication. We identified however during this work a global and structured taxonomy of these features which could be of interest.

A conversational service has the following fundamental characteristics :

- it is structured in three main temporally sequential steps : the establishment, the execution and the release

- it involves at least two principals one of which is the initiator; we name participants the other ones 
We can organize the existing features in three main functions :

- Presentation concerns the various ways informations can be exchanged (input/output) between a principal and the conversational service through her device

- Authorization identifies various conditions to be met in order to allow or interrupt a particular step of the service together with the verifying process of these conditions and its reporting
- Leg handling determines the different ways to influence the exchange of signaling information between the service and a principal during the three steps

We can identify the main characteristics of the existing features as indicated in the table below. Usually supplementary services are often expressed with standardized acronyms. Due to lack of place, we cannot provide the associated glossary; when necessary we advise the reader to consult respectively the two following references: CS-SS [1] and IMS-SS [5].

TABLE I : TAXONOMY OF CS-SS AND IMS-SS

\begin{tabular}{|c|c|c|c|c|c|c|c|c|c|c|c|c|}
\hline & \multicolumn{4}{|c|}{ Establishment } & \multicolumn{4}{|c|}{ Execution } & \multicolumn{4}{|c|}{ Release } \\
\hline & \multicolumn{2}{|c|}{ CS-SS } & \multicolumn{2}{|c|}{ IMS-SS } & \multicolumn{2}{|c|}{ CS-SS } & \multicolumn{2}{|c|}{ IMS-SS } & \multicolumn{2}{|c|}{ CS-SS } & \multicolumn{2}{|c|}{ IMS-SS } \\
\hline & Initiator & Participant & Initiator & Participant & Initiator & Participant & Initiator & Participant & Initiator & Part. & Initiator & Part. \\
\hline Presentation & $\begin{array}{l}\text { CLIR } \\
\text { COLP }\end{array}$ & $\begin{array}{l}\text { CLIP } \\
\text { COLR } \\
\text { CNAP }\end{array}$ & $\begin{array}{l}\text { OIR } \\
\text { TIP } \\
\text { AOC } \\
\text { CAT }\end{array}$ & $\begin{array}{l}\text { OIP } \\
\text { TIR } \\
\text { AOC } \\
\text { CRS }\end{array}$ & $\begin{array}{l}\text { AoCI } \\
\text { AoCC }\end{array}$ & $\begin{array}{l}\text { AoCI } \\
\text { AoCC }\end{array}$ & $\mathrm{AOC}$ & $\mathrm{AOC}$ & & $\begin{array}{l}\text { AoCI } \\
\text { AoCC }\end{array}$ & $\mathrm{AOC}$ & $\mathrm{AOC}$ \\
\hline Authorization & $\begin{array}{l}\text { CUG } \\
\text { BAOC } \\
\text { BOIC } \\
\text { BOIC- } \\
\text { exHC }\end{array}$ & $\begin{array}{l}\text { CUG } \\
\text { BAIC } \\
\text { BAIC- } \\
\text { Roam } \\
\text { ACR }\end{array}$ & OCB & $\begin{array}{l}\text { MCID } \\
\text { ACR } \\
\text { ICB }\end{array}$ & & & 3PTY & $\begin{array}{l}\text { MCID } \\
\text { 3PTY }\end{array}$ & & & & MCID \\
\hline Leg Handling & $\begin{array}{l}\text { eMLPP } \\
\text { UUS } \\
\text { CCBS }\end{array}$ & $\begin{array}{l}\text { eMLPP } \\
\text { CD } \\
\text { CFU } \\
\text { CFB } \\
\text { CFNR } \\
\text { CFNRc } \\
\text { UUS }\end{array}$ & $\begin{array}{l}\text { CCBS } \\
\text { CONF } \\
\text { CCNR }\end{array}$ & $\begin{array}{l}\text { CFU } \\
\text { CFB } \\
\text { CFNR } \\
\text { CFNL } \\
\text { CD } \\
\text { CFNRc } \\
\text { FA }\end{array}$ & $\begin{array}{l}\text { CW } \\
\text { HOLD } \\
\text { ECT } \\
\text { MPTY } \\
\text { UUS }\end{array}$ & $\begin{array}{l}\text { CW } \\
\text { HOLD } \\
\text { ECT } \\
\text { MPTY } \\
\text { UUS }\end{array}$ & $\begin{array}{l}\mathrm{CW} \\
\text { HOLD } \\
\text { ECT }\end{array}$ & $\begin{array}{l}\mathrm{CW} \\
\text { HOLD } \\
\text { ECT }\end{array}$ & UUS & UUS & & \\
\hline
\end{tabular}

It is now clear, based on the variety of service ideas and use-cases exemplified in the previous section, that adding context awareness to communication services can vastly improve their usefulness as well as their ease of use. Nonetheless, several issues are raised by this new approach, including privacy issues, architectural choices, implementation within an operator network of context data processing facilities, or standardization issues.

Privacy is a very real concern to take into account when dealing with context aware services and applications. Besides somewhat idealistic voices which denounce the "big brother syndrome", there is indeed a growing awareness among users that their private life is more and more made transparent to private corporations, with no clear rules to ensure basic rights are respected. As a consequence, for any context aware service to become widely used, its provider has to make considerable effort to ensure and demonstrate its benefits on the one hand, and its rules of play with respect to private data on the other hand. It is understood that some context data are more sensitive to privacy concerns, and that some application classes are better perceived than others. Besides, telecom operators, having a long standing commercial relationships with their subscribers, may be in a good position to act as a trusted party with respect to context data, and they must focus on how to nurture this relationship and how to increase this trust. It is generally accepted that these goals are best reached by providing valuable services in return, and by allowing users to explicitly control their data within the services. Control includes granting access, controlling the level of accuracy, sharing patterns and withdrawal policies [12].

An important aspect of deploying context aware services is related to architecture of the context management system. Many research efforts have been made in this area [12], and we envision that a hybrid approach is a viable one [14], i.e. one where some components are embedded within the core operator network while others can be deployed at the edges, even within the mobile terminals. Some well-known service enablers have already received extensive scrutiny, and they are now widely deployed components, e.g. location management, availability \& presence enablers. There is now a requirement to generalize the management of situational data in a carefully 
designed way. This is specially the case for next generation mobile networks (4G / LTE) where the low latency combined with the high throughputs offered make it eventually possible to gather real-time, multi-dimensional, contextual data about almost any user registered within the network. We are convinced now is the proper time to design a comprehensive context management scheme dedicated to such a mobile network, which is a very important issue for the relevant standardization bodies.

The origin and nature of the context data is also a matter for careful thinking and implementation choices. Actually, two broad categories of contextual data may be thought of: one is "raw data" coming from physical sensors or low-level monitoring services, and the other is "high-level data" resulting from advanced computation such as automated learning, inference or multiple sources consolidation [18]. Depending on the service, data from one category or the other may be required, and this has a strong impact on the feasibility of the related service, since sophisticated data may not be easily available for any user at any time (due to processing costs, required infrastructure, but also in some times because of the time it may take to gradually construct the data, as is the case for automated learning or collaborative filtering techniques).

\section{CONCLUSION}

In this paper, we have proposed to revisit classical telecommunication services by exploring the context awareness dimension. Doing so, we have shown a great potential for renewed services, which become more efficient, more personalized, probably easier to use and more consistent. However, actually implementing these services is a matter for further thinking, because some implementation choices such as service architecture or GUI are of paramount importance for the actual rendering and deserve a lot of attention. The user interface for instance is a critical point for the acceptability of the services, and recent advances such as voice interaction (e.g. Apple's SIRI) may well be key to more natural modes of operation, taking into account that the contextual dimension may well add complexity to the service command.

A similar axis for further work is to adopt the point of view of the operator when designing context-aware services. In the previous sections, we have developed a restrictive view, completely focused on the improvement of the quality of experience of the user, without taking into account the impact of our approach: the resource occupation ratio which has consequences on the financial aspects of the services. But a different study needs to be done which must clearly show the benefits and the cost of introducing context in the business model of such services.

On another level, we argue that the whole lifecycle of the services can be the object of context-aware redesign, and that network management processes, from the point of view of the operator, should be revisited using context.

Extrapolating on our approach, we suggest that the methodology exposed in this paper is general enough to be implemented when designing new context-aware services. A possible approach could be to iterate on a novel service specification, by taking into account trends one to four, in turns, as a way to aid injecting context awareness within the service specification.

Finally, having based our work on standards from the 3GPP consortium, we are considering contributing back to the standardization process, by advocating to turn to context awareness as a major exploration opportunity, for the next evolution steps of the telecommunication services.

\section{REFERENCES}

[1] 3GPP TS 22.004 - General on supplementary services - V11.0.0 - 2011.

[2] ITU-T Q.730 - ISDN User Part Supplementary Services - 1999.

[3] ETSI GSM 02.04 - General on supplementary services - 1995.

[4] ETSI TS 181002 - Multimedia Telephony with PSTN/ISDN simulation services - V1.1.1 - 2006

[5] 3GPP TS 22.173 - Multimedia Telephony Service and supplementary services - V.12.0.0 - 2011.

[6] GSMA PRD IR.92 - IMS Profile for Voice and SMS. 3.0 - 2010.

[7] D. Norman, The Design of Everyday Thing, Ed. Doubleday Currency, 1989.

[8] K.J. Turner, and G.A. Campbell, "Goals and Conflicts in Telephony", 10th International Conference on Feature Interactions (ICFI 2009), 1112 june 2009, Lisbon, Portugal.

[9] RCS-e - Advanced Communications: Services and Client Specification, Version 1.2.1, 16 December 2011, GSMA.

[10] 3GPP TR 22.973 - IMS Multimedia Telephony Service and supplementary services - V.11.0.0 - 2011.

[11] 3GPP TS 23.292 - IMS centralized services - V.11.2.0 - 2012.

[12] F. Toutain, A. Bouabdallah, R. Zemek, C. Daloz, "Interpersonal contextaware communication services", IEEE Communications Magazine, January 2011 , vol. $49, \mathrm{n}^{\circ} 1$, pp. 68-74, 2011.

[13] [13] 3GPP TS 23.011 - Technical realization of supplementary services V.10.0.0 - 2011.

[14] A. Bouabdallah, F. Toutain, M. Szczerbak, J-M. Bonnin, "On the Benefits of a Network-Centric Implementation for Context-Aware Telecom Services", in Proc. 15th Int. Conf. Intelligence in Next Generation Networks (ICIN 2011), 4-7 Oct. 2011, Berlin.

[15] 3GPP TS 24.604 - Communication Diversion (CDIV) using IP Multimedia (IM) Core Network (CN) subsystem; Protocol specification - V.11.2.0 - 2012.

[16] 3GPP TS 23.067 - enhanced Multi-Level Precedence and Pre-emption service (eMLPP) - V10.0.0 - 2011.

[17] X. Jiang, J. A. Lindsay, "Modeling Privacy Control in Context-aware Systems", IEEE Pervasive Computing, 2002, Vol. 1, pp.59-63.

[18] H. Erdogan, A. Ereilet, H. Abut, "Experiments on Decision Fusion for Driver Recognition", Advances for In-Vehicle and Mobile Systems, Ed. Springer Verlag, 2007, pp.1-9.

[19] Xinguo Yu, "Approaches and principles of fall detection for elderly and patient", HealthCom 2008. 10th International Conference on e-health Networking, Applications and Services, 2008, pp.42-47.

[20] ETSI TS 181002 - Multimedia Telephony with PSTN/ISDN simulation services - V2.2.5 - 2007. 